\title{
Primary cutaneous cribriform carcinoma: report of six cases with clinicopathologic data and immunohistochemical profile
}

Background: Primary cutaneous cribriform carcinoma (PCCC) is a rare and under-recognized variant of sweat gland carcinoma, characterized by anastomosing tubules and solid nests producing a sieve-like appearance.

Methods: Six cases of PCCC were clinically, histopathologically and immunophenotypically studied.

Results: All cases showed an unencapsulated, nodular dermal tumor composed of solid and cribriform nests, cords and tubules embedded within a desmoplastic stroma. Some tubules showed micropapillary projections reminiscent of tubular adenoma, and intraluminal thin bridges resembling adenomatoid tumor. One case showed a predominantly solid component. Only two cases showed very focal decapitation secretion. Tumor cells expressed CK7, CK5/6, EMA, CEA, S-100, BerEP4 and c-kit and were negative for cytokeratin 20, estrogen receptor/progesterone receptor (ER/PR), androgen receptor and GCDFP-15. Calponin, p63 and smooth muscle actin (SMA) showed absence of a myoepithelial layer.

Conclusions: Contrary to the original reports, our series shows that PCCC may be predominantly solid with only a minor cribriform component, and expresses c-kit and S-100 which may potentially lead to the misdiagnosis of adenoid cystic carcinoma. Immunohistochemical stains may aid in distinction from tubular adenoma. Current data suggest a favorable outcome, as metastatic disease has not been reported to date.

Keywords: adenoid cystic carcinoma, adenomatoid, adnexal, cribriform, papillary eccrine adenoma, tubular adenoma

Arps DP, Chan MP, Patel RM, Andea AA. Primary cutaneous cribriform carcinoma: report of six cases with clinicopathologic data and immunohistochemical profile.

J Cutan Pathol 2015; 42: 379-387. (C) 2015 John Wiley \& Sons A/S. Published by John Wiley \& Sons Ltd
David P. Arps' ${ }^{1}$, May P. Chan ${ }^{1,2}$, Rajiv M. Patel ${ }^{1,2}$ and Aleodor A. Andea ${ }^{1,2}$

1 Department of Pathology, University of Michigan, Ann Arbor, MI, USA, and ${ }^{2}$ Department of Dermatology, University of Michigan, Ann Arbor, MI, USA

Aleodor A. Andea, MD,

Department of Pathology, University of Michigan Health System, Medical Science I, 1301

Catherine St., 5336 Med Sci I 5054, Ann Arbor, Ml 48109, USA

Tel: +7347644460

Fax: +734764 4690

e-mail: andeaa@med.umich.edu

Accepted for publication February 16, 2015 


\section{Arps et al.}

Primary cutaneous cribriform carcinoma (PCCC) is a rare variant of sweat gland carcinoma with unique histopathologic features, first described in 1998 by Requena et al. ${ }^{1}$ In this initial report, five patients presented with solitary skin nodules, most commonly affecting the extremities. Histopathologically, PCCC is characterized by anastomosing nests of neoplastic cells that form cribriform spaces, imparting a sieve-like appearance. Requena et al. regarded PCCC as an apocrine neoplasm based on the observation of decapitation secretion in the neoplastic cells. Following two case reports, ${ }^{2,3}$ Rütten et al. ${ }^{4}$ published the largest series to date of 26 cases, which supported Requena's original clinicopathologic impression and contributed to the evidence that PCCC behaves in an indolent fashion.

We present our experience with six additional cases of PCCC. Our cases highlight a spectrum of histomorphology not described in the original report. We also detail an expanded immunohistochemical characterization of PCCC not previously reported. Finally, a discussion on its differential diagnosis, potential diagnostic pitfalls and clinical considerations is provided.

\section{Material and methods}

This series was compiled from cases seen in consultation at the University of Michigan as well as the personal slide collections of AAA and MPC. Clinical information was obtained from contributing clinicians as well as a search of the patient's medical record where available.

The following immunohistochemical stains were performed on four available cases of PCCC: CK5/6 (predilute: mouse monoclonal D5/16B4), S-100 (predilute: rabbit polyclonal), EMA (predilute: mouse monoclonal, E29), ER (predilute: rabbit monoclonal SP1), PR (predilute: rabbit monoclonal 1E2), calponin (predilute: rabbit monoclonal EP798Y), smooth muscle actin (SMA) (predilute: mouse monoclonal 1A4) and p63 (predilute: mouse monoclonal 4A4) from Ventana Medical Systems (Tucson, AZ, USA); CK7 (1/100; mouse monoclonal OV-TL 12/30), CK20 (1/200: mouse monoclonal Ks20.8), c-kit (1/100: rabbit monoclonal YR145) and BRST-2 (predilute; rabbit monoclonal EP1582Y) from Cell Marque (Rocklin, CA, USA); BerEp4 (1/100: mouse monoclonal) and CEA (1/1000: rabbit polyclonal) from Dako (Glostrup, Denmark). Staining was performed on formalin-fixed paraffin embedded sections using the Ventana
Benchmark Ultra automated immunostainer according to standard protocols validated by the Immunoperoxidase Laboratory within the Department of Pathology at (removed for review). Selected immunohistochemical stains were also performed on a representative case of tubular adenoma retrieved from the department files.

\section{Results}

Clinicopathologic data is summarized in Table 1 . Of the six cases studied, genders were equally represented. The average age at presentation was 43-year-old (range: 31-61 years). Tumors were limited to the extremities, including three from the arm and three from the leg. Clinical diagnosis was available for three cases and included: tender epidermal inclusion cyst, dermatofibroma and a mobile nodule. All patients underwent conservative excision with negative margins. Pathologic size ranged 4-12 mm (average: $6.5 \mathrm{~mm}$ ). Sentinel lymph node biopsies were performed in two cases, both of which were negative. Follow-up data was available in two cases with no evidence of recurrence at 16 and 22 months, respectively.

From a histopathologic perspective, the six cases of PCCC showed a unifying constellation of features. All cases showed a partially circumscribed, unencapsulated, dermal nodule composed of epithelial nests with lumen formation set in a desmoplastic stroma (Fig. 1). In four cases the tumor showed a pushing border into the subcutaneous fat; the remaining two cases were limited to the dermis. Many nests anastomosed or interconnected with neighboring nests. Invariably, neoplastic lumina and intraluminal bridges were composed of markedly attenuated epithelium producing a sieve-like appearance at low magnification. These sieve-like spaces varied in size throughout the same tumor, ranging from small to quite dilated (Fig. 2A). Small intraluminal micropapillae were also be appreciated. Scattered eosinophilic secretions were present in a minority of lumina.

The tumor nests were composed of epithelioid cells with round to polygonal, hyperchromatic nuclei, occasional small nucleoli and scant to moderate amount of dense eosinophilic cytoplasm. The cells lining the luminal spaces and producing the intraluminal bridges ranged from cuboidal to markedly flattened, with elongated nuclei. Areas of attenuation were one or several layers thick, however there was no appreciable myoepithelial layer. Decapitation secretion was 
Primary cutaneous cribriform carcinoma

Table 1. Clinicopathologic features of PCCC

\begin{tabular}{|c|c|c|c|c|c|c|}
\hline \multicolumn{7}{|l|}{ Clinical features } \\
\hline Age & 41 & 32 & 35 & 59 & 61 & 31 \\
\hline Gender & $\mathrm{F}$ & $\mathrm{F}$ & M & $\mathrm{F}$ & M & M \\
\hline Location & Leg & Elbow & Leg & Arm & Arm & Leg \\
\hline Clinical impression & EIC, tender & NA & NA & $N A$ & Mobile nodule & $\mathrm{DF}$ \\
\hline SNL status (if performed) & & $0 / 1$ & & & & $0 / 2$ \\
\hline Recurrence & NTD & NA & NTD & $N A$ & NA & NA \\
\hline \multicolumn{7}{|l|}{ Histopathology } \\
\hline Pathologic size (mm) & 6 & 5 & 5 & 4 & 7 & 12 \\
\hline Solid/tubular\% & $40 / 60 \%$ & $10 / 90 \%$ & $50 / 50 \%$ & $95 / 5 \%$ & $60 / 40 \%$ & $5 / 95 \%$ \\
\hline Desmoplastic stroma & Yes & Yes & Yes & Yes & Yes & Yes \\
\hline Single cells at periphery & Yes & Yes & Yes & Yes & Yes & Yes \\
\hline Lymphoid aggregates & Yes & No & Yes & Yes & Yes & Yes \\
\hline Decapitation secretion & Focal & No & No & No & No & Focal \\
\hline Lymphovascular/neural invasion & No & No & No & No & No & No \\
\hline
\end{tabular}

F, female; M, male; NA, data not available; SLN, sentinel lymph node; NTD, none to date; EIC, epidermal inclusion cyst; DF, dermatofibroma.
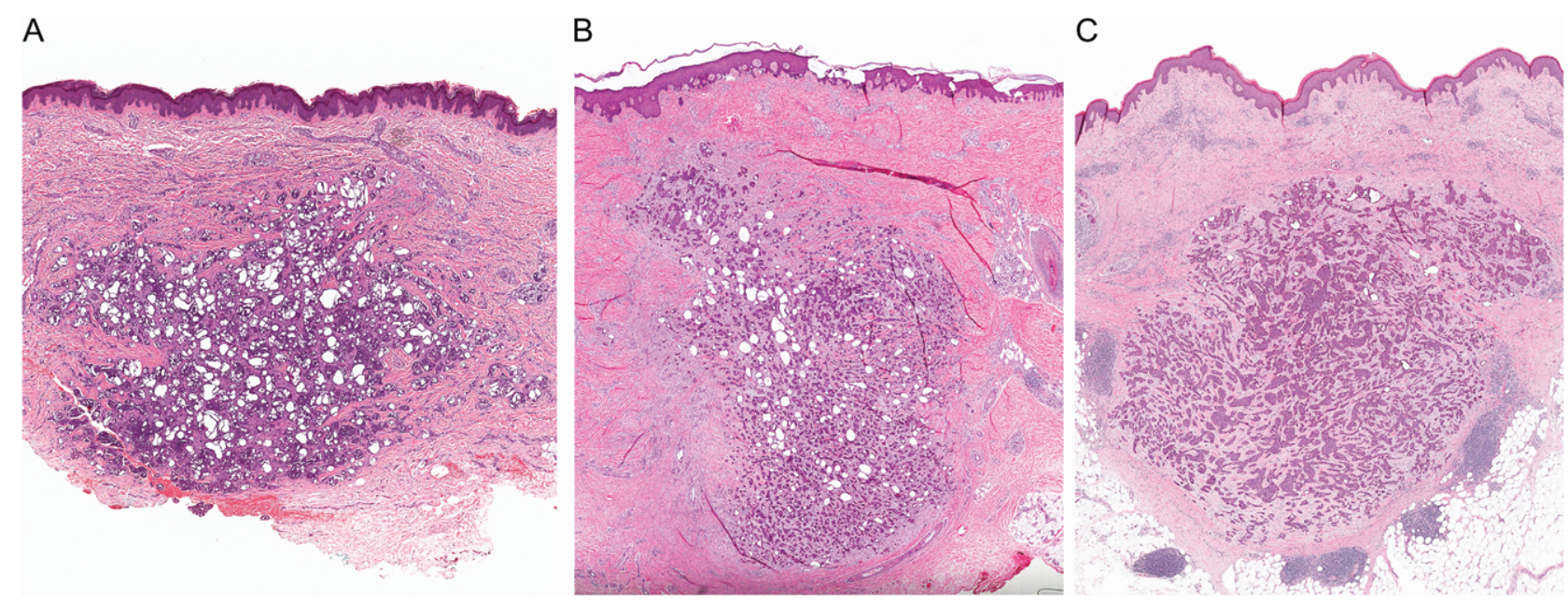

Fig. 1. Primary cutaneous cribriform carcinoma. On low magnification, the tumor forms a relatively well-circumscribed, unencapsulated dermal nodule. The majority of primary cutaneous cribriform carcinomas (PCCCs) appear sieve-like because of numerous dilated cystic spaces [A: hematoxylin and eosin (H\&E), ×20]. However, PCCC may also show areas of solid growth admixed with cystic spaces $(\mathrm{B}: \mathrm{H} \& \mathrm{E}, \times 15)$, or less commonly be composed of predominantly solid nests $(\mathrm{C}: \mathrm{H} \& \mathrm{E}, \times 15)$.

focally present in two cases and absent in the remaining cases (Fig. 2B). In situ lesions, such as apocrine hyperplasia or ductal carcinoma in situ, were absent. Infrequent mitoses were identified (range: $1-3$ mitoses per 10 fields at $\times 400$ ) as well as occasional, scattered single cell necrosis.

The ratio of solid nests compared to lumen formation varied dramatically among the six examples. In one case, $95 \%$ of tumor nests showed lumen formation with the remaining $5 \%$ showing solid growth. In contrast, another case was predominantly solid with only $5 \%$ of the tumor nests forming characteristic dilated lumens with attenuated lining epithelium (Fig. 3). With limited lumen formation, this case did not show the characteristic sieve-like, cribriform appearance. The remaining cases contained a roughly equal proportion of tubular and solid components.

On close inspection of the periphery of the neoplasms, rare scattered infiltrative single cells were appreciated in all cases. These cells were embedded in the desmoplastic stroma associated with the tumor, and did not extend beyond $1 \mathrm{~mm}$ from the main tumor mass. No lymphovascular or perineural invasion was identified. There was no connection between the neoplastic nests and the overlying, unremarkable epidermis or existing adnexal structures. Lymphoid aggregates were present at the periphery of five cases.

Immunohistochemical stains were performed on four cases of PCCC and one case of tubular adenoma with results summarized in Table 2. The tumor cells were strongly and diffusely 


\section{Arps et al.}

A

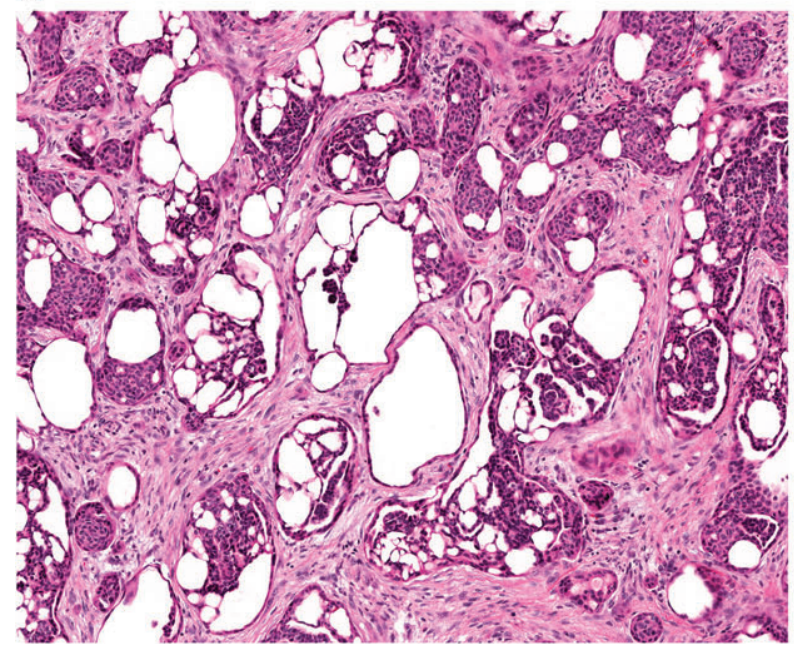

B

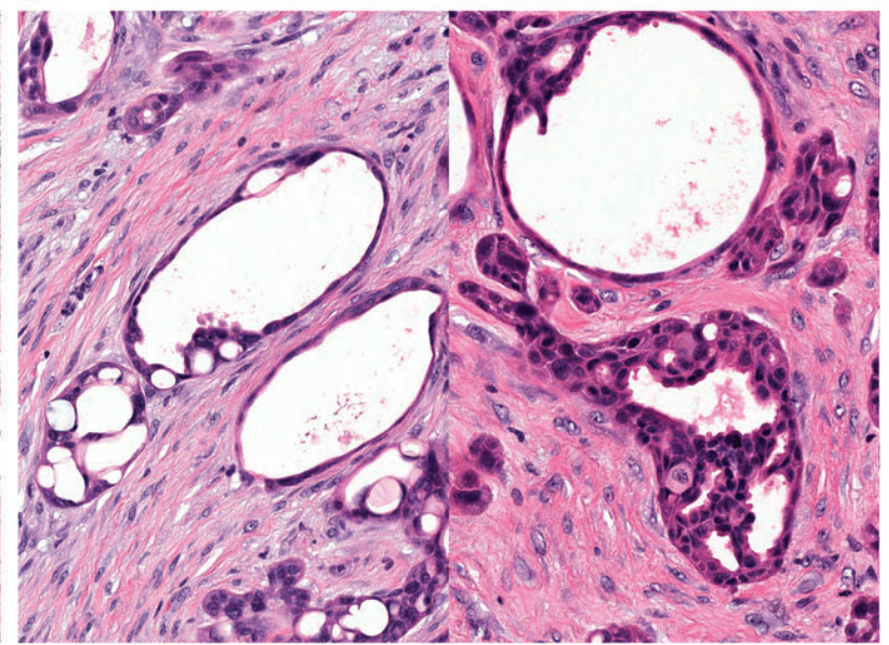

Fig. 2. Histomorphologic features of primary cutaneous cribriform carcinoma. Dilated cystic spaces with markedly attenuated epithelium. Within some of those spaces are intraluminal micropapillae and intraluminal bridges imparting a cribriform growth pattern [A: hematoxylin and eosin $(\mathrm{H} \& \mathrm{E}), \times 100 \mathrm{x}]$. Focal areas suggestive of decapitation secretion were present in two of six cases of PCCC (B: H\&E, 200x).

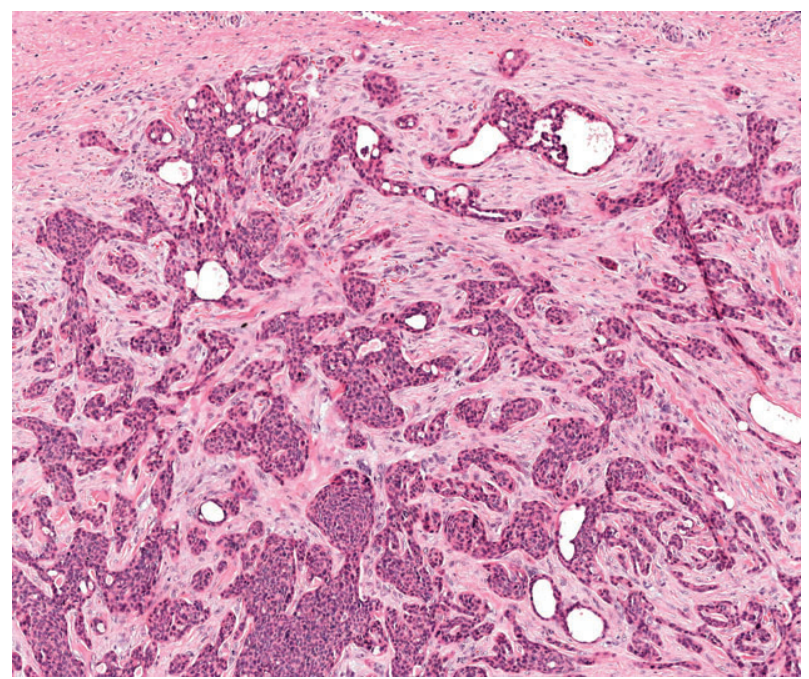

Fig. 3. Predominantly solid variant of primary cutaneous cribriform carcinoma with focal attenuated luminal epithelium, bridging and micropapillae [hematoxylin and eosin (H\&E), $\times 80]$.

immunoreactive for CK5/6 and CK7 while negative for CK20. S-100 was also positive in all cases in a patchy to diffuse pattern (Fig. 4A). CEA and EMA staining demonstrated luminal positivity. C-kit (CD117, Fig. 4B) and BerEp4 were also positive. BRST-2 and ER/PR were negative in all cases in which these stains were performed. Calponin and SMA highlighted myofibroblasts within the desmoplastic stroma. Staining for p63 highlighted rare, scattered neoplastic cells within the tumor nests. There was no evidence of a myoepithelial layer on either hematoxylin
Table 2. Immunohistochemical profile of primary cutaneous cribriform carcinoma (PCCC) and tubular adenoma (TA)

\begin{tabular}{|c|c|c|c|c|c|}
\hline & PCCC & & & & TA \\
\hline CK5/6 & + & NA & + & + & + \\
\hline CK7 & + & + & + & + & + \\
\hline CK20 & - & - & NA & - & - \\
\hline $\mathrm{S} 100$ & + & + & + (patchy) & + & $+($ rare $)$ \\
\hline CEA & +luminal & +luminal & $N A$ & +luminal & NA \\
\hline EMA & +luminal & +luminal & NA & +luminal & NA \\
\hline C-kit & + & $N A$ & + & + (patchy) & + (patchy) \\
\hline BerEp4 & + & NA & + & + & + \\
\hline BRST-2 & - & NA & - & - & - \\
\hline ER/PR & - & - & $N A$ & - & NA \\
\hline Calponin & +stroma & NA & NA & +stroma & +myoepi \\
\hline SMA & +stroma & $N A$ & +stroma & +stroma & +myoepi \\
\hline p63 & $+($ rare $)$ & NA & $+($ rare $)$ & $+($ rare $)$ & +myoepi \\
\hline
\end{tabular}

NA, data not available; myoepi, myoepithelial layer;,$+>50 \%$ of tumor cells are positive; patchy, $25-50 \%$ of tumor cell are positive; rare, $<1 \%$ of tumor cells are positive.

and eosin-stained sections or by immunohistochemistry with p63, SMA and calponin (Fig. 5). The control case of tubular adenoma included in the study showed rare S-100 positive cells ( $<1 \%$ of cells), patchy immunoreactivity for c-kit (25-50\% of cells), and a conspicuous myoepithelial layer positive with p63, SMA and calponin was noted (Fig. 6).

\section{Discussion}

PCCC is a rare variant of sweat gland carcinoma. The clinical presentations and outcomes of 
A

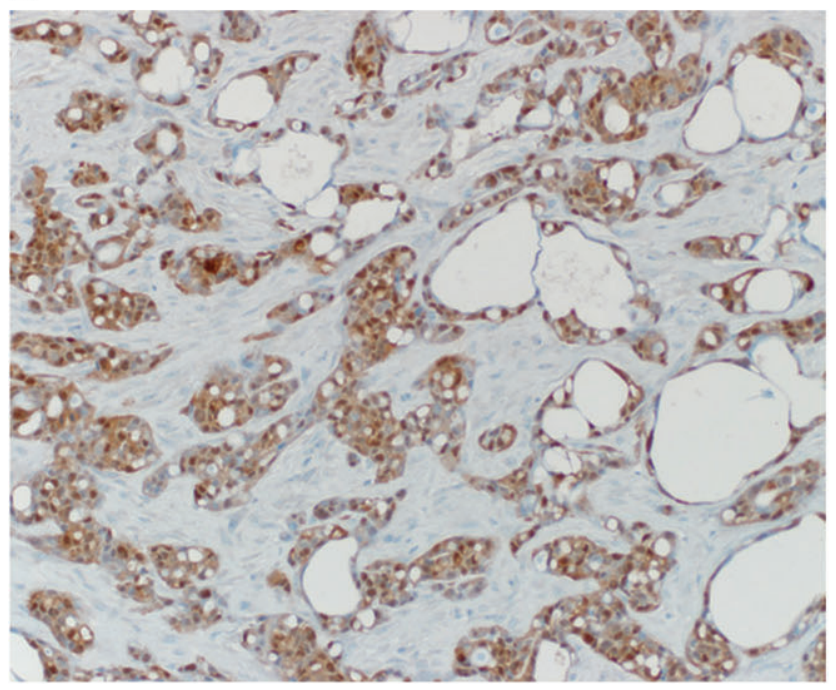

B

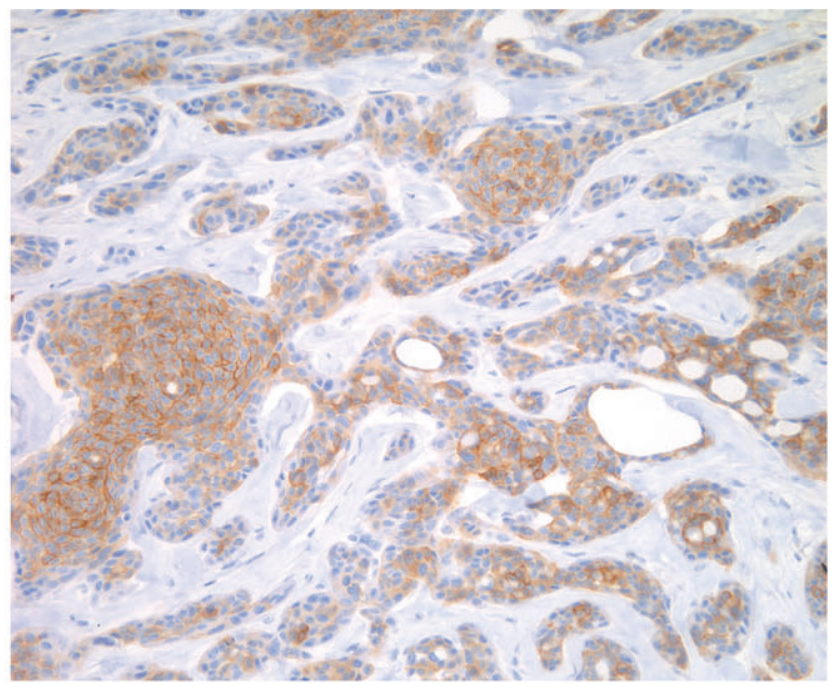

Fig. 4. Primary cutaneous cribriform carcinoma shows diffuse immunostaining for S-100 (A: $\times 200)$ and membranous c-kit $($ B: $\times 200)$ positivity.

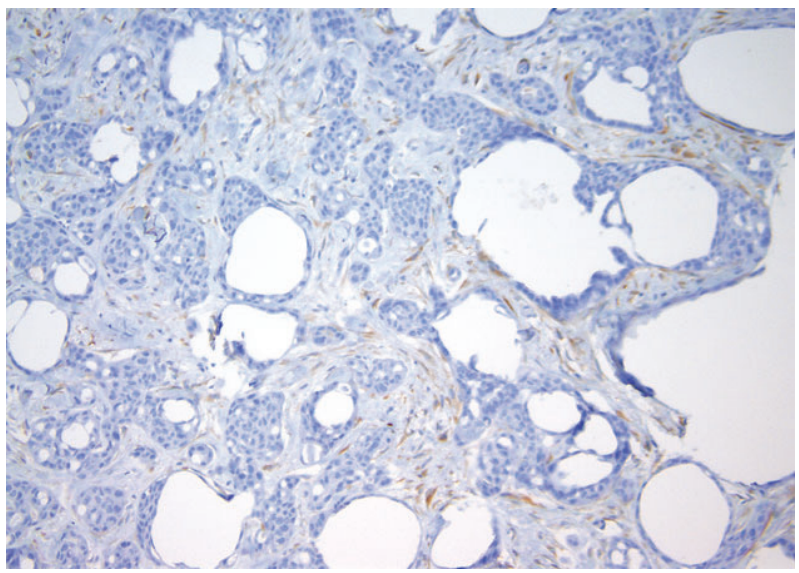

Fig. 5. Primary cutaneous cribriform carcinoma is negative for a myoepithelial layer by calponin immunostain $(\times 200)$.

PCCCs from this study and previous reports are summarized in Table 3. The neoplasm presents as a solitary dermal nodule without overlying epidermal changes. Aggregating all data available on PCCC, ${ }^{1-4}$ women are more frequently affected (2: 1 female to male ratio), with an average age of 47 years. To date there has been no report of recurrence or metastases. Requena et al. reported persistence at the site secondary to incomplete excision. ${ }^{1}$ Unlike the original description of $\mathrm{PCCC}^{1}$, in which 'the entire neoplasm has a cribriform pattern,' our series contains one case that is predominantly solid. Recognition of this spectrum of histomorphology is important, as other carcinomas such as adenoid cystic carcinoma (ACC) may also show a predominantly solid pattern. ACC has a propensity for perineural invasion, recurrence and metastasis in some cases,${ }^{5-10}$ none of which have been observed in PCCC. In our one case with predominantly solid nests (Fig. 3), few foci toward the periphery displayed a more characteristic appearance including attenuated luminal epithelium and thread-like intraluminal bridges, supporting the diagnosis of PCCC.

Several authors considered PCCC to be an apocrine adenocarcinoma based on the finding of decapitation secretion. ${ }^{1,2}$ Apocrine adenocarcinoma is a broad category, encompassing several defined entities such as digital papillary adenocarcinoma, syringocystadenocarcinoma papilliferum and malignant apocrine mixed tumor. ${ }^{11}$ However, when histopathologic criteria are not met for these entities, the diagnosis of apocrine carcinoma not-otherwise-specified (NOS) may be rendered, thus resulting in a heterogeneous group of carcinomas. In such cases, decapitation secretion or cytoplasmic zymogen granules are generally regarded as evidence of apocrine differentiation. Additionally, in situ precursor lesions may be observed in these tumors, ${ }^{12-16}$ although a larger series suggests that these are uncommon. ${ }^{17}$ In situ lesions are analogous to those routinely seen in breast pathology, namely, columnar cell change, usual ductal hyperplasia, atypical ductal hyperplasia and ductal carcinoma in situ. ${ }^{11}$ Apocrine differentiation may be appreciated in these precursor lesions. Staining for myoepithelial markers such as calponin, p63 and SMA can help distinguish in situ lesions from the invasive component. 


\section{Arps et al.}

A

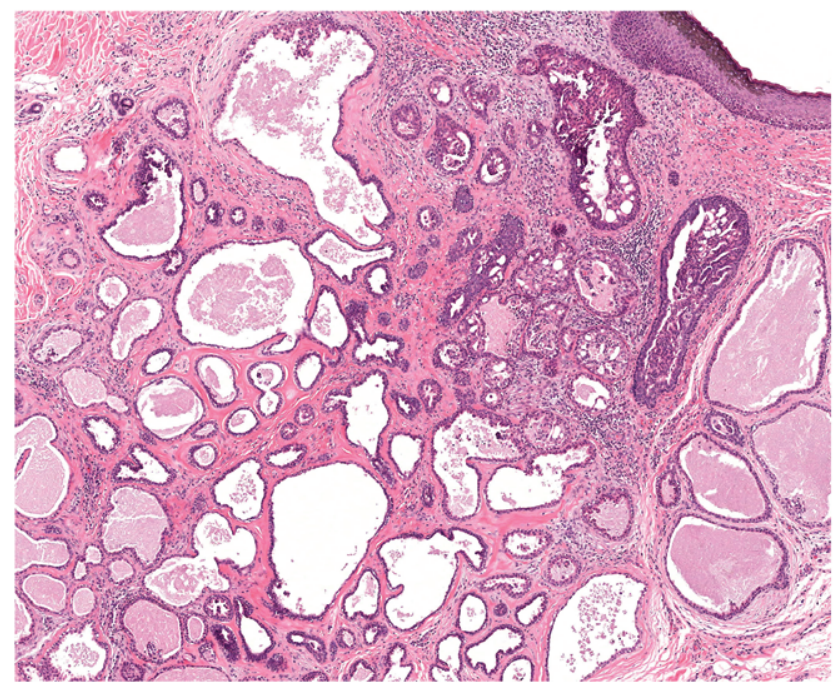

B

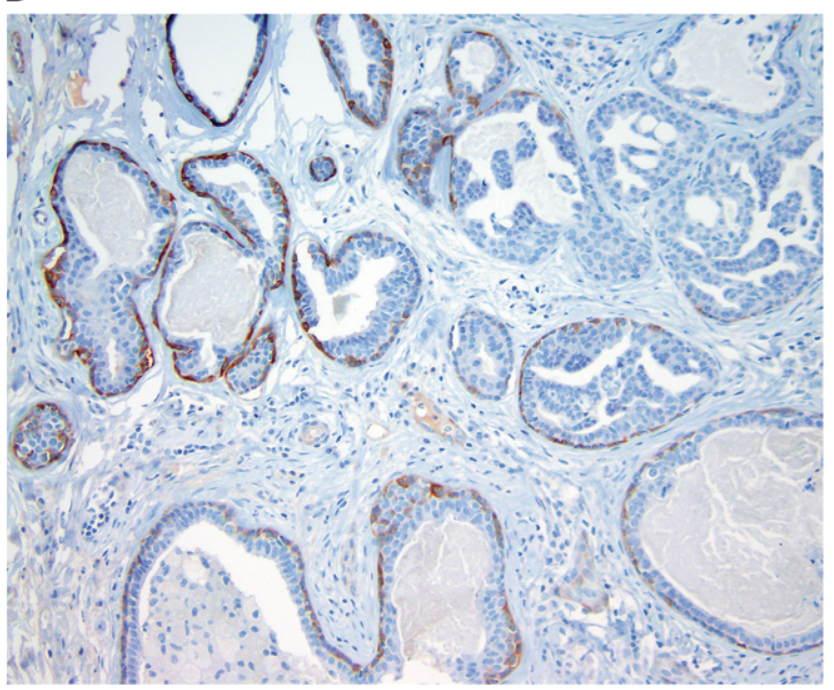

Fig. 6. Tubular adenoma may have similar histopathologic findings to primary cutaneous cribriform carcinoma, including dilated cystic spaces with attenuated epithelium, micropapillae, and focal intraluminal bridging. The stroma is paucicellular and fibrous (A: hematoxylin and eosin $(\mathrm{H} \& \mathrm{E}), \times 100)$. Calponin immunostain highlights the myoepithelial layer surrounding nests in tubular adenoma (B; 200x).

Table 3. Clinical presentations and outcomes of PCCC reported in the literature and in this study

\begin{tabular}{|c|c|c|c|c|}
\hline & Sex (number of cases) & Average age (range) & Location & Available follow up \\
\hline Requena et al. ${ }^{1}$ & * & 44 years $(20-55)$ & $\begin{array}{l}3 \text { lower ext. } \\
1 \text { upper ext. } \\
1 \text { pubis }\end{array}$ & $\begin{array}{c}2 \text { cases persisted at site secondary } \\
\text { to incomplete excision. No } \\
\text { metastases. }\end{array}$ \\
\hline Rütten et al. ${ }^{4}$ & $\begin{array}{l}F(19) \\
M(7)\end{array}$ & 47.8 years $(23-77)$ & $\begin{array}{c}10 \text { lower ext. } \\
9 \text { upper ext. } \\
4 \text { trunk } \\
1 \text { head } \\
1 \text { neck }\end{array}$ & $\begin{array}{l}13 \text { cases with no recurrence or } \\
\text { metastases after re-excision. }\end{array}$ \\
\hline Adamski et al. $^{2}$ & M & 37 years & lower ext. & No recurrence or metastases. \\
\hline Fernandez-Flores et al. $^{3}$ & $\mathrm{~F}$ & 62 years & lower ext. & No recurrence or metastases. \\
\hline Current report & $\begin{array}{l}F(3) \\
M(3)\end{array}$ & 43 years $(31-61)$ & $\begin{array}{l}3 \text { lower ext. } \\
3 \text { upper ext. }\end{array}$ & $\begin{array}{c}2 \text { cases with no recurrence or } \\
\text { metastases. }\end{array}$ \\
\hline Summary of reported data & $\begin{array}{l}\mathrm{F}(23 ; 67 \%) \\
\mathrm{M}(11 ; 32 \%)\end{array}$ & 47 years $(20-77)$ & $\begin{array}{c}18 \text { lower ext. }(47 \%) \\
13 \text { upper ext. }(34 \%) \\
4 \text { trunk }(10 \%) \\
1 \text { head }(3 \%) \\
1 \text { neck }(3 \%) \\
1 \text { pubis }(3 \%)\end{array}$ & $\begin{array}{c}2 \text { cases persisted at site secondary } \\
\text { to incomplete excision. No cases } \\
\text { with recurrence or metastases. }\end{array}$ \\
\hline
\end{tabular}

F, female; M, male; ext., extremity.

*Reported as 'mainly women.'

Some authors have questioned the apocrine derivation of PCCC. ${ }^{3}$ In our series, we appreciated only very focal decapitation secretion in two cases. Close inspection of the tumors and the surrounding adnexal structures in conjunction with immunohistochemistry showed no in situ lesions. Similarly, no previous reports of PCCG have described an in situ component to our knowledge. Furthermore, the lack of staining for BRST-2 (GCDFP-15) in our series also provides some evidence against apocrine differentiation. ${ }^{18,19}$ Unlike most other apocrine carcinomas which have a propensity to occur in the axilla or anogenital region, PCCC does not appear to follow this distribution but instead shows a predilection for the extremities. The true derivation of PCCC therefore remains uncertain.

Our study presents a new finding of membranous c-kit (CD117) immunoreactivity in PCCC 


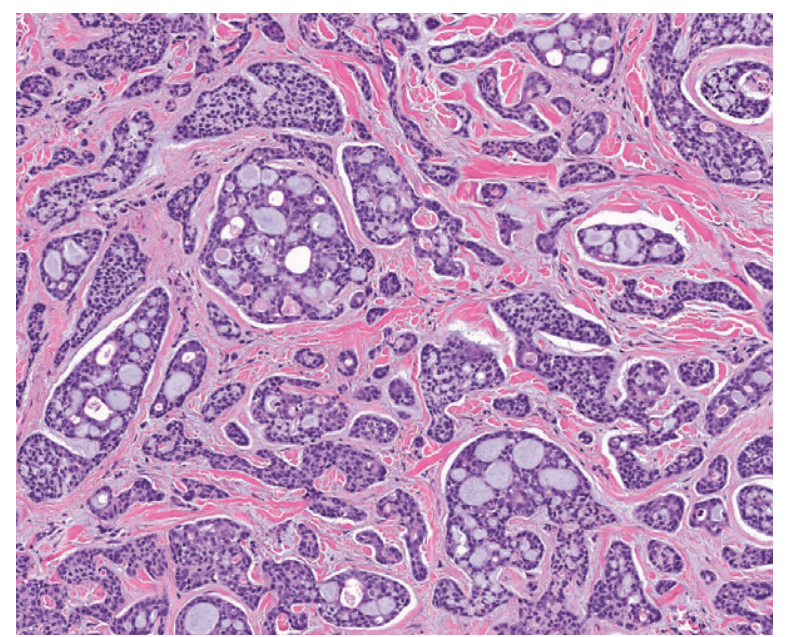

Fig. 7. Adenoid cystic carcinoma is arranged in discrete lobules and demonstrates basophilic mucinous material within cystic spaces [hematoxylin and eosin (H\&E), $\times 100$ ].

(Fig. 4B). This raises a potential diagnostic pitfall, namely confusion with ACC, either primary cutaneous or metastatic, which is also positive for c-kit. ${ }^{5,20}$ ACC typically shows a mixture of cribriform and solid growth patterns. However, unlike PCCC, ACC is poorly circumscribed with infiltrative growth. ACC is arranged in discrete lobules, demonstrates basophilic mucinous material within cystic spaces, and lacks the characteristic marked epithelial attenuation and intraluminal micropapillae seen in PCCC (Fig. 7). The finding of perineural invasion should raise concern for ACC, as ACC is notoriously neurotropic, whereas perineural invasion has not been reported in PCCC. ACC is also typically S-100 positive..$^{5,11,21}$ The majority of previous reports have described negative staining for S-100 in $\mathrm{PCCC}^{2-4} \mathrm{How}^{-}$ ever, we found S-100 to be positive in all our cases of PCCC in which the stain was performed (Fig. 4A), ranging from diffuse (three cases) to patchy (one case).

In ours and other investigators experience, ${ }^{22}$ PCCC most closely resembles tubular adenoma. The World Health Organization (WHO) classifies both papillary eccrine adenoma and tubular apocrine adenoma as tubular adenoma. ${ }^{23}$ As PCCC is currently regarded as a low-grade carcinoma, distinction from tubular adenoma is clinically relevant. The microscopic features of tubular adenoma are variable and may overlap with those of PCCC (Fig. 6). For example, tubular adneoma may be composed of dilated cystic spaces lined by markedly attenuated epithelium, similar to those observed in PCCC. It may also display micropapillae and focal intraluminal bridging. In contrast to PCCC which is invariably set in a desmoplastic stroma, tubular adenoma typically shows a paucicellular fibrous stroma. Interestingly, decapitation secretion in tubular adenoma is far more conspicuous than in PCCC. Key to the distinction is the recognition of a myoepithelial layer in tubular adenoma, which is lacking in PCCC. In areas of markedly attenuated epithelium this may be difficult to appreciate, however tubular adenoma typically has scattered glands in which a continuous outer myoepithelial layer can be appreciated on hematoxylin and eosin-stained sections. In smaller biopsies, however, recognition of myoepithelium and other subtle features may be challenging. In such cases, immunohistochemical stains for myoepithelium such as p63, calponin or
A

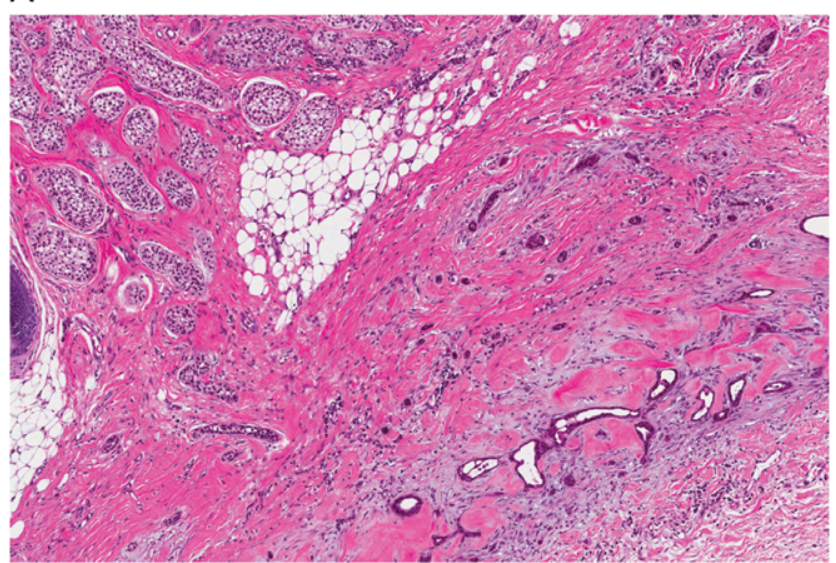

B

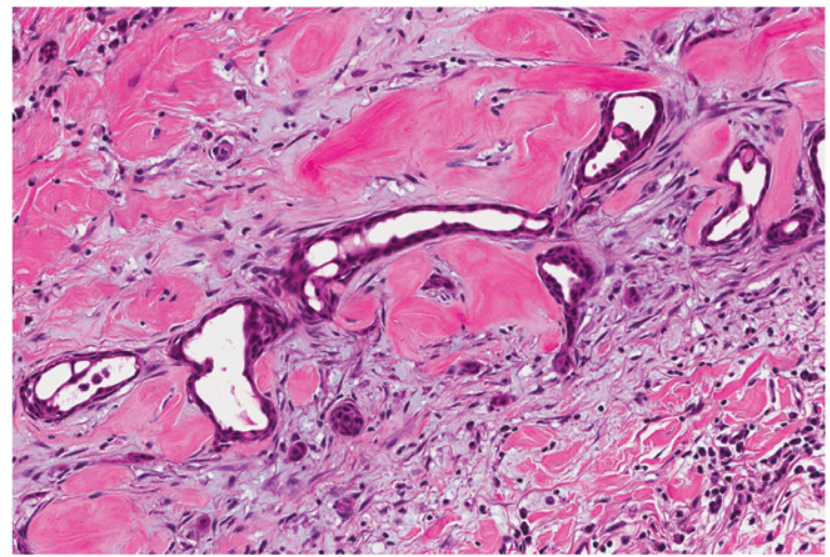

Fig. 8. Eccrine carcinoma of the scalp with clear cell change in the dermis and a second population of deeper infiltrative glands demonstrating many of the features of PCCC [A: hematoxylin and eosin (H\&E), $\times 50$ ]. Nests are composed of dilated spaces lined by markedly attenuated with occasional thread-like intraluminal bridges and micropapillae (B: H\&E, $\times 100)$. 
SMA may be helpful. The tumor nests in PCCC consistently lack a myoepithelial layer, with myoepithelial immunostains highlighting only a subset of stromal myofibroblasts (Fig. 5). In contrast, the glands of tubular adenoma are surrounded by a single layer of myoepithelial cells highlighted by either p63, calponin or SMA (Fig. 6). Even in areas of marked attenuation, at least a subset of the glands will exhibit myoepithelial staining. In our experience, calponin or p63 immunostaining show less background staining of stromal myofibroblasts and thus may be easier to interpret.

Another pathologic mimic of PCCC is the mesothelial-derived adenomatoid tumor. ${ }^{24}$ Interestingly, Crippa et al. reported a skin tumor closely resembling adenomatoid tumor, which was negative for mesothelial markers by immunohistochemistry. ${ }^{25}$ As Kazakov et al. noted, this may very well have been an example of PCCG. ${ }^{22}$ One may also consider the possibility of cribriform-patterned metastasis from other sites such as the breast, salivary gland, colon, prostate, uterus or ovaries. Close inspection would probably reveal microscopic features divergent from PCCC, such as a cribriform appearance but lacking thread-like intraluminal bridges, or the presence of high-grade cytomorphology or dirty necrosis. Immunohistochemical stains such as BRST-2, ER, PR, CK20, CDX-2, PSA and PAX-8 may be helpful in supporting the diagnosis of metastasis and in determining the site of origin.
It is also important to note that tumors with features of eccrine carcinoma or syringomatous carcinoma (both which may be variants of microcystic adnexal carcinoma) may occasionally show focal areas with features of PCCC. ${ }^{11}$ We have recently encountered a consultation such a case of eccrine carcinoma of the scalp with clear cell change with a second population of deeper infiltrative glands showing many of the features of PCCC (Fig. 8) The tumor nests were composed of dilated spaces lined by markedly attenuated epithelium with occasional thread-like intraluminal bridges and micropapillae. However, the overall pattern of infiltration is not in keeping with PCCC, this example of eccrine carcinoma was broadly infiltrative, not a nodular proliferation. Whereas clear cell change has been described previously in eccrine carcinoma, ${ }^{26,27}$ it is not a feature of PCCC.

In summary, we report the unique clinicopathologic features of PCCC. As opposed to the original reports, our cases showed that PCCC may be predominantly solid with only a minor cribriform component. We also demonstrated that PCCC expresses c-kit and S-100 by immunohistochemistry, a potential pitfall in its differentiation from ACC. Tubular adenoma can be distinguished from PCCC by the presence of a myoepithelial layer around the tumor nests. PCCC is currently regarded as a low-grade carcinoma and appears to behave in an indolent fashion; treatment is complete excision.

\section{References}

1. Requena L, Kiryu H, Ackerman AB. Neoplasms with apocrine differentiation. Philadelphia: Lippencott-Raven, 1998.

2. Adamski H, Le Lan J, Chevrier S, Cribier B, Watier E, Chevrant-Breton J. Primary cutaneous cribriform carcinoma: a rare apocrine tumour. J Cutan Pathol 2005; 32 577.

3. Fernandez-Flores A, Pol A, Juanes F, Crespo LG. Immunohistochemical phenotype of cutaneous cribriform carcinoma with a panel of 15 antibodies. Med Mol Morphol 2007; 40: 212.

4. Rütten A, Kutzner H, Mentzel T, et al. Primary cutaneous cribriform apocrine carcinoma: a clinicopathologic and immunohistochemical study of 26 cases of an under-recognized cutaneous adnexal neoplasm. J Am Acad Dermatol 2009; 61: 644.

5. Ramakrishnan R, Chaudhry IH, Ramdial $\mathrm{P}$, et al. Primary cutaneous adenoid cystic carcinoma: a clinicopathologic and immunohistochemical study of 27 cases. Am J Surg Pathol 2013; 37: 1603.

6. Chang CH, Liao YL, Hong HS. Cutaneous metastasis from adenoid cystic carcinoma of the parotid gland. Dermatol Surg 2003; 29: 775 .

7. Sanderson KV, Batten JC. Adenoid cystic carcinoma of the scalp with pulmonary metastasis. Proc R Soc Med 1975; 68: 649.

8. Kato N, Yasukawa K, Onozuka T. Primary cutaneous adenoid cystic carcinoma with lymph node metastasis. Am J Dermatopathol 1998; 20: 571.

9. Chang SE, Ahn SJ, Choi JH, Sung KJ, Moon KC, Koh JK. Primary adenoid cystic carcinoma of skin with lung metastasis. J Am Acad Dermatol 1999; 40: 640.

10. Chu SS, Chang YL, Lou PJ. Primary cutaneous adenoid cystic carcinoma with regional lymph node metastasis. J Laryngol Otol 2001; 115: 673.

11. Kazakov DV, Michal M, Kacerovska D, McKee PH. Cutaneous adnexal tumors. Philadelphia: Lippencott Williams \& Wilkins, 2012

12. Weinreb I, Bergfeld WF, Patel RM, Ghazarian DM. Apocrine carcinoma in situ of sweat duct origin. Am J Surg Pathol 2009; 33: 155 .
13. Castelli E, Wollina U, Anzarone A, Morello V, Tomasino RM. Extramammary paget disease of the axilla associated with comedo-like apocrine carcinoma in situ. Am J Dermatopathol 2002; 24: 351.

14. Castro CY, Deavers M. Ductal carcinoma in-situ arising in mammary-like glands of the vulva. Int J Gynecol Pathol 2001; 20: 277.

15. Obaidat NA, Awamleh AA, Ghazarian DM. Adenocarcinoma in situ arising in a tubulopapillary apocrine hidradenoma of the peri-anal region. Eur J Dermatol 2006; 16 : 576.

16. Yoshida A, Kodama Y, Hatanaka S, Takasaki T, Kuriwaki K, Yoshida H. Apocrine adenocarcinoma of the bilateral axillae. Acta Pathol Jpn 1991; 41: 927.

17. Robson A, Lazar AJ, Ben Nagi J, et al. Primary cutaneous apocrine carcinoma: a clinico-pathologic analysis of 24 cases. Am J Surg Pathol 2008; 32: 682.

18. Wick MR, Ockner DM, Mills SE, Ritter JH, Swanson PE. Homologous carcinomas of the breasts, skin, and salivary glands. A 


\section{Primary cutaneous cribriform carcinoma}

histologic and immunohistochemical comparison of ductal mammary carcinoma, ductal sweat gland carcinoma, and salivary duct carcinoma. Am J Clin Pathol 1998; 109: 75 .

19. Miyamoto T, Hagari Y, Inoue S, Watanabe T, Yoshino T. Axillary apocrine carcinoma with benign apocrine tumours: a case report involving a pathological and immunohistochemical study and review of the literature. J Clin Pathol 2005; 58: 757.

20. Mino M, Pilch BZ, Faquin WC. Expression of KIT (CD117) in neoplasms of the head and neck: an ancillary marker for adenoid cystic carcinoma. Mod Pathol 2003; 16: 1224.
21. Wick MR, Swanson PE. Primary adenoid cystic carcinoma of the skin. A clinical, histological, and immunocytochemical comparison with adenoid cystic carcinoma of salivary glands and adenoid basal cell carcinoma. Am J Dermatopathol 1986; 8: 2.

22. Kazakov DV, Plaza JA, Suster S, Kacerovska D, Michal M. Cutaneous cribriform carcinoma: a short comment. J Am Acad Dermatol 2011; 64: 599.

23. LeBoit PE, Burg G, Weedon D, Sarasin AE. World Health Organization classification of tumours. Pathology and genetics of skin tumours. Lyon, France: IARC Press, 2006.

24. Hes O, Perez-Montiel DM, Alvarado Cabrero I, et al. Thread-like bridging strands: a morphologic feature present in all adenomatoid tumors. Ann Diagn Pathol 2003; 7: 273

25. Crippa S, Di Bella C, Faravelli A. Skin adnexal neoplasm closely resembling adenomatoid tumor: a unique occurrence. Int J Surg Pathol 2006; 14: 177.

26. Sánchez Yus E, Requena Caballero L, Garcia Salazar I, Coca Menchero S. Clear cell syringoid eccrine carcinoma. Am J Dermatopathol 1987; 9: 225.

27. Wong TY, Suster S, Nogita T, Duncan LM, Dickersin RG, Mihm MC Jr. Clear cell eccrine carcinomas of the skin. A clinicopathologic study of nine patients. Cancer 1994; 73: 1631. 